\title{
Targeting Karyotypic Complexity and Chromosomal Instability of Cancer Cells
}

\author{
Anna V. Roschke* and llan R. Kirsch \\ Genetics Branch, Center for Cancer Research, National Cancer Institute, Building NNMC8, Room \\ 5101, Bethesda, MD 20889-5105, USA
}

\begin{abstract}
Multiple karyotypic abnormalities and chromosomal instability are characteristic features of many cancers that are relatively resistant to chemotherapeutic agents currently used in the clinic. These same features represent potentially targetable "states" that are essentially tumor specific. The assessment of the chromosomal state of a cancer cell population may provide a guide for the selection or development of drugs active against aggressive and intractable cancers.
\end{abstract}

\section{Keywords}

Aneuploidy; chromosomal instability; cancer; anticancer drugs; NCI-60; karyotypic abnormalities; CIN; chromosomal instability phenotype

\section{INTRODUCTION}

Most cancers have an abnormal chromosomal content characterized by clonal changes in chromosomal structure and number. In addition, cancer cell populations very often show nonclonal cell-to-cell chromosomal heterogeneity. This heterogeneity is a marker of ongoing chromosomal instability in cancers - accelerated rates of changes in chromosome structure, gains and losses of chromosome pieces or whole chromosomes.

Cancer karyotypes can be distinguished by the level of their complexity, varying from the presence of only one abnormal chromosome in the diploid set of chromosomes up to very complex karyotypes with almost every chromosome structurally rearranged and/or present in abnormal quantity (http://cgap.nci.nih.gov/Chromosomes/Mitelman). Karyotypic complexity is reflected in the aneuploidy of cancer cells. Aneuploidy is an unbalanced number of chromosomes or chromosome segments, and is a result of past or present structural and numerical chromosomal instability and selection.

The level of karyotypic heterogeneity varies from tumor to tumor, and may differ during stages of cancer progression. Complexity and heterogeneity of cancer karyotypes do not strictly correlate. For instance, the most karyotypically complex cancer cell lines are not necessarily the most heterogeneous [1] and (http://www.ncbi.nlm.nih.gov/sky/).

Chromosomal instability can mediate the evolution of cancer cell populations under selection pressure [2-4]. Chromosomal aberrations tend to be more numerous in malignant tumors than in benign ones, and aneuploidy and chromosomal instability are associated with 
poorer prognoses, and aggressive clinical and distinctive histopathologic features [5-12]. Therefore, multiple karyotypic abnormalities and chromosomal instability are particular hallmarks of many cancers that are relatively resistant to long-term control by current chemotherapeutic agents.

Aneuploidy and chromosomal instability represent two phenotypes or "states" of cancer cells. Other comparable "states" might include base pair hypermutability. Just as a case will be made in what follows that the "state" of karyotypic complexity or instability may provide a tumor specific target for anti cancer therapy, there have already been preliminary explorations of how targeting the tumor specific state of mismatch repair defectiveness may be an avenue for anti cancer therapeutic development [13-16]. The specific genes amplified, deleted, or reconfigured can distinguish a tumor from the normal cells from which it arose and represent potential targets for cancer treatment. But, in addition, the very fact of aneuploidy and karyotypic instability represent "states" that are essentially tumor specific. Potentially these states could provide targets for anticancer therapy.

\section{ANEUPLOIDY AND CHROMOSOMAL INSTABILITY AS CANCER PHENOTYPES}

\section{Constitutional Aneuploidy and Cellular Fitness}

Constitutional aneuploidy causes developmental abnormalities and reduced organismal and cellular fitness in all species where it has been studied (reviewed in [17]). Systematic studies of segmental aneuploidies in Drosophila showed that under $0.5 \%$ of the haploid genome is tolerated as heterozygous deficient, whereas flies that are triploid for parts of the genome are viable, but regardless of the identity of a triploid segment they have lower viability, reduced body size, and developmental abnormalities that become more pronounced as the size of trisomic segment increases; extensive trisomies are lethal [18]. In humans, fetuses with autosomal monosomies and multiple trisomies are extremely rare among spontaneous abortions, indicating that these conditions lead to gamete or early embryonic lethality [19]. Only three autosomal trisomies exist that survive to birth (trisomies of chromosome 13, 18 and 21), with only Down syndrome patients surviving until adulthood.

Aneuploid cells have reduced proliferation rates and exhibit metabolic alterations. Primary foreskin fibroblasts of trisomy 21 patients proliferate more slowly than euploid control cells [20]. Mouse cells engineered to be trisomic for specific chromosomes exhibit a proliferation delay and impaired immortalization capacity [21]. Studies on yeast strains carrying one or two additional chromosomes show that aneuploidy leads to cell proliferation disadvantages in Saccharomyces cerevisiae [22], and hampers cell proliferation in Schizosaccharomyces pombe [23].

Recent findings on the effects of aneuploidy per se on cell physiology suggest that aneuploidy puts significant stress on the cell. Mouse trisomic cells exhibited metabolic alterations with glutamine uptake and ammonium production being increased; glucose uptake was altered as well [21]. Saccharomyces cerevisiae strains carrying additional chromosomes have an increased glucose uptake, and a decrease in biomass produced per glucose molecule. This may indicate that more energy is being used to degrade proteins and induce mechanisms shielding the cell from the effects of excess proteins [22]. They also exhibit a gene expression pattern characteristic of a general stress response and increased sensitivity to compounds that interfere with protein synthesis, turnover and folding. 


\section{Aneuploidy and Cancer}

How aneuploidy contributes to tumorigenesis is not yet understood. It appears, that aneuploidy alone is not sufficient for carcinogenesis. Most children with Down's syndrome do not develop leukemia. In the presence of widespread aneuploidies due to mosaic variegated aneuploidy cancers are developed only in $<50 \%$ of cases. Constitutional aneuploidy, therefore, requires cooperating genetic somatic events to progress to neoplasia, but in most cases these events have not been investigated (reviewed in [24]).

Copy-number alterations of cancer genomes have been extensively studied by chromosomal $\mathrm{CGH}$ and array CGH. Specific patterns of genomic gains and losses are characteristic of different cancer types [10-11, 25-28], and the level of aneuploidy usually increases with cancer progression [5-6, 11]. High levels of aneuploidy in cancer samples are predictors of poor prognosis $[6-7,12]$. A gene expression signature associated with a higher level of segmental aneuploidy also predicts poor prognosis in six cancer types [29]. Moreover, this gene expression signature correlates with resistance to paclitaxel and sensitivity to carboplatin in ovarian cancers [30].

Evidence suggests that pathways mediating chromosomal stability are associated with paclitaxel sensitivity. Depleting spindle assembly checkpoint proteins promotes resistance to paclitaxel [31]. Kinome siRNA screens reveal regulators of mitotic checkpoint and chromosomal instability as common mediators of resistance to paclitaxel in vitro [32].

Unlike point mutations that affect only a relatively small number of genes during tumorigenesis, a gain or loss of a chromosome can affect transcription of hundreds of genes simultaneously and disturb a large array of cellular processes [28, 33]. Based on observations of detrimental consequences of aneuploidy in yeast and mice, a hypothesis was proposed that aneuploidy is actually a barrier to tumorigenesis [21, 34, 35]. Given the deleterious effects of aneuploidy, how can aneuploidy promote tumorigenesis? Why are most tumors aneuploid? To reconcile these views, it was suggested that the very events that cause aneuploid cells to proliferate slowly, the cellular imbalances caused by aneuploidy and the stresses associated with it, are responsible for selective pressures that promote tumorigenesis [17].

\section{Chromosomal Instability in Cancer Cells}

It has long been considered that genomic instability is an integral component of neoplasia $[36,37]$. Instability is reflected in heterogeneity seen within individual tumors and cancer cell lines. Originated from a single cell, at the time of clinical diagnosis many tumors are composed of mixtures of genetically diverse subclones [38].

There are several distinct forms of genomic instability. Integrity of the genome and the fitness and viability of the cells is ensured by a network of genome surveillance mechanisms and DNA repair pathways. These pathways include mismatch repair, homologous recombination, non-homologous end-joining, base excision repair, and translesion synthesis. Genomic integrity is also maintained by the cellular machinery with checkpoints that control equal chromosome segregation prior to cell divisions, ensuring that the correct complement of chromosomes is passed to daughter cells. Accordingly, there are multiple routes of disruption leading to increased instability of the genome.

Two of the best characterized forms of instability are microsatellite instability (MIN, changes in short tandem DNA repeat sequences) and chromosomal instability (CIN, gains and losses of whole chromosomes) [39, 40]. Between them, CIN is the most prevalent form of instability. The term CIN refers only to numerical chromosomal instability - gains and losses of chromosomes - but two types of chromosomal instability are seen in cancer cells: 
structural and numerical chromosomal instability. Cancer cells experience persistent gains and losses of whole chromosomes as well as changes in chromosomal structure, including reciprocal and nonreciprocal translocations, inversions, deletions, duplications and amplifications [41-43].

Defining CIN as a process where rates of gains and losses of chromosomes may vary [39] led to the realization that ongoing instability represents a persistent defect in chromosome segregation during mitosis that continuously scrambles the chromosomal content of cancer cells. It was found that tumors with ongoing chromosomal instability carry a poor prognosis in different cancer types $[8,11,12]$. Chromosomal instability generates the necessary chromosome diversity in the tumor cell population and transcriptome diversity to allow for environment-facilitated clonal expansion and clonal evolution of cancer cell populations $[10$, 44].

\section{Causes of CIN}

The precise molecular mechanisms that underlie CIN in tumor cells remain unclear, Given the complexity of mitosis there are multiple routes by which mitotic fidelity can be lost and by which cells might gain or lose chromosomes (reviewed in [45]).

a) Defects in Mitotic Checkpoint Signaling-A weakened mitotic checkpoint may allow cells to enter anaphase in the presence of unattached or misaligned chromosomes. Mitotic checkpoint dysfunction has been extensively studied in mouse models (reviewed in [46-48]). So far, conventional gene knockouts have been constructed for four core SAC (spindle assembly checkpoint) components (Mad2, Mad1, BubR1 and Bub3), and four modulators (Chfr, Rae1, Nup98 and CenpE) [34, 49-53]. In addition, hypomorphic alleles that express dramatically reduced levels of BUB1 and BUBR1 have also been generated [54, 55]. Whereas complete loss of these gene products results in early embryonic lethality, heterozygous and hypomorphic mice are viable and fertile. In all cases, mice with genetically reduced levels of mitotic checkpoint components have an increased level of aneuploidy and CIN in mouse embryonic fibroblasts (MEFs) and tissues. Although aneuploid animals with reduced levels of BUB1, BUBR1, BUB3, RAE1 or both RAE1 and NUP98 fail to display an increase in spontaneous tumorigenesis, these mice are prone to carcinogen-induced tumors [54, 56-58], suggesting that aneuploidy does not initiate cancer in these mouse models, but rather drives tumor formation in cases in which mutations at oncogenic or tumor suppressor loci have already increased the potential for cellular transformation.

CIN genes when defective can promote or suppress tumorigenesis depending on the genetic background, and they affect only a few tissues. Mice with a reduced level of the SAC regulator and kinetochore motor CENP-E display an increase in the frequency of spontaneous lymphomas and benign lung tumors. CENP-E heterozygous animals lacking the tumor suppressor gene p19/ARF have a decreased incidence of tumors relative to controls $[59,60]$. Bub1 insufficiency predisposes $\mathrm{p}^{33^{+-}}$mice to thymic lymphomas and $\mathrm{Apc}^{\mathrm{minl}+}$ mice to colonic tumors through the loss of chromosome with non-mutated tumor suppressor allele and gain of a copy of the mutated allele. In contrast, Bub1 insufficiency has no impact on tumorigenesis in $\mathrm{Rb}^{+1-}$ mice and inhibited prostatic intraepithelial neoplasia formation in Pten $^{+\downarrow}$ mice [61].

An extensive search for mitotic checkpoint defects in human cancers has uncovered very infrequent mutations of mitotic checkpoint components, and more frequent altered expression of mitotic checkpoint genes BUB1, BUBR1, BUB3, MAD1, MAD2 (reviewed in [62]). 
b) Cohesion Defects-If sister chromatid cohesion is lost prematurely or persists during anaphase, chromosomes can be missegregated. To identify mechanisms that lead to aneuploidy in cells, genes that have putative functions in guarding against chromosome missegregation were systematically sequenced in a panel of aneuploid colorectal cancers [63]. Ten of the 11 mutations identified were in genes that directly contribute to sister chromatid cohesion, indicating that defects in the machinery that controls sister chromatid cohesion might promote aneuploidy. Consistently, overexpression of separase or securin, two key regulators that control the loss of chromatid cohesion, promotes aneuploidy and cellular transformation [64-65]. However, those studies only explain the cause of CIN in a minority of tumor cells.

c) Multiple Centrosomes-Cells that possess more than two centrosomes might form multiple spindle poles during mitosis. If this defect is not corrected then a multipolar division might occur, resulting in the production of highly aneuploid and often nonviable daughter cells. Increased centrosome number correlates with aneuploidy and is a common characteristic of cancers. However, centrosomes in multipolar spindles often cluster into two groups to allow cells to divide in a bipolar fashion [66-68]. Centrosome clustering may increase the frequency of incorrect kinetochore microtubule attachments (such as merotelic attachments (see next section)). Extra centrosomes are therefore capable of driving chromosome missegregation through a mechanism that is independent of multipolar divisions. It was recently shown that transient defects in spindle geometry in cancer cells, such as those caused by supernumerary centrosomes, elevate the incidence of merotelic attachments, indicating that some cancer cells with CIN have excessive rates of formation of attachment errors $[69,70]$.

d) Merotelic Attachment-One kinetochore can attach to microtubules from both poles of the spindle and form a merotelic attachment. If these attachments persist into anaphase then lagging chromatid pairs might be missegregated or excluded from both daughter cells during cytokinesis. Unlike other mal-orientations, merotely evades SAC detection [71]. Instead, merotelic correction is regulated by the Aurora B kinase to orchestrate release of inappropriately oriented microtubules, permitting replacement by properly oriented microtubules $[72,73]$.

Cancer cells with CIN have excessive rates of formation of merotelic attachments [69, 70] and diminished capacity to correct them [74-76]. It was shown that relatively minor perturbations in kinetochore microtubule attachment dynamics are sufficient to disturb attachment stability required for faithful chromosome segregation, and restoration of kinetochore microtubule attachment dynamics leads to the suppression of CIN [74-77]. Thus, there is not just one gene responsible for CIN, given that imbalances in any of a number of proteins might be sufficient to disturb normal kinetochore microtubule dynamics.

Chromosome missegregation compromises the proliferation of diploid cells, indicating that additional changes that permit the propagation of nondiploid cells must combine with elevated chromosome missegregation rates to generate aneuploid cells with CIN [75].

\section{Chromosomal Instability Phenotype}

Chromosomal instability is a multi-layered phenotype comprising an increased predisposition to a missegregation of chromosomes during mitosis and to an aberrant repair of DNA breaks, as well as a survival state specifically adapted to the constant reshuffling of the genome [78].

Relationships between chromosomal instability and cellular functions have not been studied in detail so far, but some features associated with chromosomal instability in cancer cells 
have been identified [79]. Correlations between karyotypic heterogeneity (as a marker of underlying numerical chromosomal instability) and gene-expression profiles of the panel of cancer cell lines (NCI-60 panel) pointed to specific cellular processes associated with chromosomal instability in cancer cells.

"Gene Ontology" (GO) analysis of the distribution of 360 genes whose expression correlated with numerical heterogeneity indicated that cell communication and signal transduction, cell adhesion, motility, and migration, response to wounding and inflammatory response, negative regulation of cell proliferation, and DNA replication are the main biological processes associated with numerical heterogeneity $(\mathrm{NH})$ of the chromosomal content in the cancer cells. Moreover, these genes fell into two groups based on their positive or negative correlation coefficients, showing a striking difference between them. Genes, expression of which were positively correlated with NH, fell into GO categories such as cell communication and signal transduction, including cell surface receptor-linked signal transduction, cell adhesion, locomotion, motility, and migration, development, morphogenesis, and differentiation, response to wounding, and inflammatory response. Products of these genes were associated with extracellular matrix and extracellular space, plasma membrane, and cytoskeleton and were involved in the focal adhesion pathway, cytokine-cytokine receptor interaction, regulation of the actin cytoskeleton, JAK-STAT signaling pathways, cell communication, and ECM-receptor interaction pathways [79].

Genes whose expression negatively correlated with NH fell into totally different GO categories: cellular metabolism, nucleic acid metabolism, regulation of transcription, DNA replication, response to DNA damage stimulus, DNA repair, chromosome organization and biogenesis, DNA packaging, unwinding and replication initiation, and base-excision repair. Products of those genes serve as transcription regulators, involved in nucleic acid binding, linked to ATP-ase activity, and associated with the cell cycle regulation pathway. They localize on intracellular organelles and are, for the most part, found in the nucleus, chromosome/chromatin, or nuclear envelope.

Regulators of the mitotic cell cycle checkpoint (MAD2 and BUB3) as well as a component of the APC/C anaphase promoting complex/cyclosome, APC4, are found among the genes whose expression is negatively correlated with $\mathrm{NH}$. Correlations do not imply causative relationships; however, it is possible that the decreased level of mitotic checkpoint components could be the basis of mitotic checkpoint relaxation leading to increased gains and losses of chromosomes. This supports already existing assumptions that a compromised mitotic checkpoint leads to accelerated rates of chromosomal instability.

The expression of genes involved in DNA damage checkpoints (CHK1, CHK2, H2AX, RAD21, XRCC5, DDB1) and DNA rereplication prevention (BCCIP, BRCA2, CDT1, MCM2-7, cyclin B2) negatively correlates with $\mathrm{NH}$ as well. The expression levels of genes involved in DNA packaging, chromosome condensation, and kinetochore formation $(\mathrm{H} 3$ histone, H1FX, H2AX, H2AZ, TOP1, RCC1, RCC2, SMARCA5, RCBTB1, CENPC1, ZWINT) are also relatively down-regulated in cancer cells with higher levels of chromosomal instability compared to cancer cells with a lower levels of instability. Chromosomal instability is associated with less effective cellular metabolism, DNA replication and transcription, DNA repair and packaging, weakness in proper chromatin condensation, and mitotic chromosome structural organization possibly owing to extensive imbalances in cellular protein composition of cells that undergo continuous gains and losses of parts of the genome.

A collective molecular portrait of the chromosomal instability phenotype in cancer cells includes relative upregulation of genes that are associated with increased motility and 
migration, epithelial-mesenchymal transition (EMT), and are critical for tumor invasion and metastasis: RhoC, fibronectin, LOX, TWIST, SNAI2, EGFR, laminins, integrins, collagens, CDC42 effector protein (Rho GTPase binding), Rho family GTPase 3, RAB, CXCL2, TGFb2, VEGFC, IL-6, IL-8, CTGF, vimentin, N-cadherin, CD44, BCAR3, protocadherins, MMP2 and MMP14, NOTCH2, SERPINE1, 2, and 8, IGFBP3 and 7, TNFAIP3, TNFRSF12A and 19, PLAUR, and SPARC. Expression of several genes that promote cell proliferation and G1 entry into the cell cycle (CCD1, EGFR, VEGFC) correlate positively with higher $\mathrm{NH}$ as well [79].

In summary, existing data suggest that the CIN phenotype is associated with the following features:

a. Changes in the cell cycle organization and coordination leading to random unequal distribution of genetic material in daughter cells;

b. Changes in metabolism and cellular functions due to genomic imbalances and alterations;

c. Intracellular mechanisms of adaptation to the consequences of genome reshuffling;

d. Extracellular mechanisms of adaptation leading to survival and selection of karyotypically unstable and aneuploid cells as part of a program of the response to tissue damage with gradual internalization of proliferative and survival signals.

\section{TARGETING ANEUPLOIDY AND CHROMOSOMAL INSTABILITY OF CANCER CELLS}

\section{Tolerance to Aneuploidy and CIN as Anticancer Targets}

Proliferation of aneuploid tumor cells with ongoing chromosomal instability suggests that there are specific adaptive mechanisms that allow tolerance of aneuploidy and CIN. Evidence of such adaptive mechanisms emerges from studies of aneuploid yeast strains. For instance, independent haploid yeast strains disomic for each of the yeast chromosomes have increased expression of genes involved in ribosome biogenesis and are sensitive to inhibitors of protein translation and protein folding [22]. The sensitivity of these strains to proteasome inhibitors seems more likely to be explained by an enhanced requirement for protein degradation in order to correct stochiometric protein imbalances [17]. If this response is consistent between aneuploid yeast and cancer cells, this supports the possibility for targeting cancer cells based on the aneuploid phenotype they possess.

Drugs that interfere with centrosome clustering mechanisms could potentially be lethal to tumor cells with multiple centrosomes, but spare normal cells. A genome-wide RNAi screen in near-tetraploid Drosophila S2 cells identified a number of genes required for centrosome clustering [80]. The classes of genes identified in this screen enabled the identification of a range of cellular processes that control organization of multipolar centrosomes: the SAC components Mad2, BubR1 (human Bub1), CENP-Meta (human CENP-E) as well as genes involved in cell polarity, actin regulation and cell adhesion. Among genes that promote binding of spindle MTs, the minus end directed kinesin motor HSET has attributes as a future therapeutic target because it appears to be essential only in cells with extra centrosomes [81].

Although several current anticancer drugs, including Taxol, can promote multipolar mitoses, none are specific for cells with multiple centrosomes [82]. Taking advantage of the tumorspecific phenotype of centrosomal clustering, a cell-based screening strategy was used to identify small molecules that inhibit centrosomal clustering and thus force tumor cells with supernumerary centrosomes to undergo multipolar mitoses, and subsequently, apoptosis. 
Screening of a relatively small but diverse natural product extract library led to the identification of griseofulvin, which induced multipolar spindles by inhibition of centrosome coalescence, mitotic arrest, and subsequent cell death in tumor cell lines but not in diploid fibroblasts and keratinocytes with normal centrosome content [83]. Following this work, 34 griseofulvin analogues were synthesized and tested as inhibitors of centrosomal clustering [84]. The most active analogues were the 2'-benzyloxy and 2'-(4-methylbenzyloxy) analogues as well as the oxime of the former with a 25 -fold increase of activity compared to griseofulvin.

\section{Screening for Potential Anticancer Agents Using "State" of Karyotypic Complexity or Instability}

Interrogation of the data-rich drug discovery panel of 60 human cancer cell lines (the NCI-60), used by the National Cancer Institute to screen compounds for anticancer activity, provided an extensive list of potential anticancer agents targeting aneuploid and chromosomally unstable cancer cells [85-87].

Detailed characterization of chromosomal alterations present in the NCI-60 panel of cancer cell lines revealed a wide variation in complexity and heterogeneity of karyotypes [1]. Every cell line in the NCI-60 panel contains karyotypic abnormalities with notable variations among the individual cell lines at the level of karyotypic complexity and heterogeneity (structural and numerical).

As a first snapshot, a 1429-drug subset of compounds was tested against the cell lines [85]. This subset was selected because each agent had been tested at least four times on all or most of the NCI-60. Most of the drugs used clinically for cancer treatment were included in this subset, along with many candidates that have reached clinical trials. Correlation analysis (with application of additional filters to minimize false-positive associations) revealed a focus group of fifty-three chemical compounds. Grouping of the 53 selected compounds based on their functional classification or chemical structure allowed the delineation of seven groups of compounds with more growth inhibitory activity against karyotypically complex and/or heterogeneous cancer cells than against karyotypically simple and homogenous ones (Table 1). These data supported the thesis that it is possible to discover potential anticancer agents based on association of their activity with a determinant of karyotypic state.

For anticancer drugs currently routinely used in the clinic this analysis did not find evidence of direct positive association between cytotoxic profiles and the variables of karyotypic state. These results suggest that the mechanisms of action of many well-known anticancer agents are most likely not associated with aneuploidy and the chromosomally unstable status of cancer cells. This is consistent with their somewhat limited utility in epithelial cancers that reside at the more karyotypically complex end of the cancer spectrum.

A much larger scale correlation analysis was performed utilizing the full set of publicly available screening data, consisting of cell-based growth inhibition data for approximately 30,000 potentially anticancer chemical compounds tested on the NCI60 cell lines [86]. The aim of this study was to identify chemical motifs and "tool" anticancer drug candidates based on their association with the karyotypic state of cancer cells. This analysis correlated karyotypic variables with drug-induced cytotoxicity measures and also applied additional filters to identify a set of compounds whose activity was associated with a cellular karyotype. With application of conservative and biologically relevant selection procedures, 13 classes of chemical compounds were identified that express more growth-inhibitory activity toward cancer cell lines with more complex and/or unstable karyotypes. 
Association between screening data organized via a self-organizing map (SOM) into target categories (anti-metabolites, tubulin interactors, alkylators, etc.) and karyotypic parameters was used to suggest distinct mechanisms of action associated with karyotypic state. The SOM anticancer maps organize the data from tested agents into regions which share the same pattern of growth inhibition, substantially reflect their molecular targets and modes of action, and provide a means of visual translation into a two dimensional map [88].

An earlier analysis of the anticancer agents in this data set found that certain regions on the SOM could be associated with putative biological mechanisms of growth inhibition. In particular, regions on the SOM were delineated that account for agents described previously in the literature as active against DNA synthesis, mitosis, membranes, xenobiotic metabolism, etc. $[89,90]$. In addition to cataloging compounds according to a mechanism of action, the results revealed an inherent interconnectedness between various cellular processes and specific growth inhibition patterns.

Projections on these maps of the compounds that have been identified as positively and significantly correlated with karyotypic parameters mainly hit a previously unexplored region in the SOM, where standard anticancer drugs are not, for the most part, present, and where mechanisms of action of chemical compounds are among the least elucidated [86]. In Fig. (1a) this is illustrated for the $\mathrm{GI}_{50}$ data vectors that are positively correlated with each of the karyotypic variables (numerical heterogeneity, numerical complexity, structural heterogeneity, and structural complexity), where each variable identified 1198, 1006, 1271, and 382 compounds, respectively. In practical terms, this result indicates that the karyotypic profiles best match the cytotoxic profiles within the subregion P3. To compare these results with those derived from current clinically relevant agents, Fig. (1b) localizes on the SOM several agents currently used as "standard of care" in cancer therapy so that their positions can be appreciated relative to the compounds showing activity determined by the karyotypic variables used in this study. At the top left, in subregions M1 and M2, reside the Vinca alkaloids (vinblastine, vindesine, and vincristine) and the taxanes (docetaxel and paclitaxel). In the bottom right of the SOM, in subregions S3 to S6, reside common clinical agents that interfere with DNA processing, such as the anthracyclines (daunorubicin, epirubicin, idarubicin, and doxorubicin), antifolates (methotrexate, raltitrexed, and pemetrexed), alkylators (oxiplatin, cisplatin, and dacarbazine), topoisomerase agents (irinotecan, amsacrine, etoposide, and teniposide), gemcitabine, 5-fluorouracil, etc. No cytotoxicity patterns from known anticancer drug classes are strongly identified by the positively correlated projections based on karyotypic variable. These findings suggest that the compounds identified as active against karyotypically complex and/or chromosomally unstable cancer cells may, indeed, represent new classes and mechanisms of action [86, 87].

Identified compounds represent an unexplored set of chemical motifs whose activities correlate with the variability of the cellular karyotypes. The 13 classes of potential agents have been delineated as motifs A to L, and are presented in the Fig. (2).

Motif A consists of cucurbitacins, a class of natural products that are thought to be antiinflammatory, possibly via actin/vimentin disruption [91, 92] and/or signal transduction modulation [93]. Another intensively studied group of molecules included in motif B are the cytochalasins. Cells treated with cytochalasins are arrested in anaphase due to actin depolymerization and a block of cytokinesis $[94,95]$. No mechanisms of action are known for the bisnaphthylcarboxamides, bis-naphthylureas, and anilinomalonyl phenylazopyrazoles, shown in motifs $C$ and D. These drugs are most closely associated with the $\mathrm{P}$ region and correlate most strongly with the numerical complexity, numerical heterogeneity, and structural heterogeneity patterns. One of the pyridinethione carbonitrile nucleosides listed as motif $\mathrm{E}$ in Table 3 is a P-glycoprotein antagonist [96]. The 
pentachlorophenyl polypeptide esters defined as motif $\mathrm{F}$ correlate specifically with the numerical complexity. One compound in this set has been identified as a potential modifier of the c-erbB2 pathway [97]. Motifs $\mathrm{G}$ to J encompassing thiazolyl coumarins, anilino/ phenoxycarboxy/phenyl-6(7)-substituted quinoxalines, 1,8-bis(5-aryloxymethyl-4anilino-1,2,4-triazol-2-yl)octanes, and 3-alkylidene-5,5-disubstituted tetrahydro-2-furanones are not associated with any known mechanism of action or target. Motif I appears in the S6 region of the map, which is colocalized with an abundance of topoisomerase inhibitor $\mathrm{GI}_{50}$ data vectors. This motif carries specificity for the structural heterogeneity karyotypic variable. The 2-substituted mercapto-3H-quinazolines listed as motif $\mathrm{K}$ and mainly found in the $\mathrm{P}$ region of the SOM were originally tested for antibacterial, antifungal, and antiacetylcholinesterase activities [98]. Subsequent studies involving these compounds have identified them as kinesin inhibitors [99]. The N-(p-(substituted azole)phenyl) benzenesulfon-amides defined as motif $\mathrm{L}$ are largely uncharacterized; however, it is interesting to note that this motif and motifs $\mathrm{E}$ and $\mathbf{J}$ are the only ones indicating specificity toward struc-tural complexity. The 1,1-dimethyl-3-phenyl-3-pyrrolidinyl/ 4-morpholinyl naphthalans (motif M) are again a relatively unexplored group of structures, although loosely related substructures have been shown to be inhibitors of thymidylate synthase [100], which is critical for DNA repair and replication.

The set of drugs that have been identified via this karyotype/drug correlation analysis provides a set of baseline compounds for further studies. A striking correlation pattern indicates that the karyotypic observables are often correlated with a relatively unexplored region on the SOM. Compounds identified in this study may target genes or pathways; however, it is important to recognize that certain agents may be active against the "state" of complexity or instability itself rather than against any specific gene product or pathway.

\section{SUMMARY COMMENTS}

Karyotypic complexity (aneuploidy) and chromosomal instability are particular features of the most aggressive cancer cells that are often relatively resistant to anticancer agents currently used in clinic. At the same time these features represent phenotypes or "states" that distinguish cancer cells from their normal counterparts. These features, therefore, provide a possibility for the screening of potential anticancer agents. Acquisition of aneuploidy or chromosomal instability seems to be detrimental for the propagation of diploid cells. There are specific adaptive mechanisms that allow tolerance of aneuploidy and chromosomal instability, and these adaptations may make cancer cells vulnerable to specific therapeutic agents. Therefore, the assessment of the chromosomal state of a cancer cell population may provide a future guide for the selection and development of drugs active against aggressive and otherwise intractable cancers.

\section{Acknowledgments}

We are grateful to W. Michael Kuehl for critical reading of the manuscript.

\section{REFERENCES}

1. Roschke AV, Tonon G, Gehlhaus KS, et al. Karyotypic complexity of the NCI-60 drug-screening panel. Cancer Res. 2003; 63(24):8634-8647. [PubMed: 14695175]

2. Nowell PC. The clonal evolution of tumor cell populations. Science. 1976; 194(4260):23-28. [PubMed: 959840]

3. Wolman SR. Cytogenetic heterogeneity: its role in tumor evolution. Cancer Genet Cytogenet. 1986; 19(1-2):129-140. [PubMed: 3455660] 
4. Nicholson JM, Duesberg P. On the karyotypic origin and evolution of cancer cells. Cancer Genet Cytogenet. 2009; 194(2):96-110. [PubMed: 19781442]

5. Ried T, Heselmeyer-Haddad K, Blegen H, et al. Genomic changes defining the genesis, progression, and malignancy potential in solid human tumors: a phenotype/genotype correlation. Genes Chromosomes Cancer. 1999; 25(3):195-204. [PubMed: 10379865]

6. Risques RA, Moreno V, Ribas M, et al. Genetic pathways and genome-wide determinants of clinical outcome in colorectal cancer. Cancer Res. 2003; 63(21):7206-7214. [PubMed: 14612515]

7. Blegen H, Will JS, Ghadimi BM, et al. DNA amplifications and aneuploidy, high proliferative activity and impaired cell cycle control characterize breast carcinomas with poor prognosis. Anal Cell Pathol. 2003; 25(3):103-114. [PubMed: 12775914]

8. Nishizaki T, Harada K, Kubota H, et al. Chromosome instability in malignant astrocytic tumors detected by fluorescence in situ hybridization. J Neurooncol. 2002; 56(2):159-165. [PubMed: 11995817]

9. Reshmi SC, Gollin SM. Chromosomal instability in oral cancer cells. J Dent Res. 2005; 84(2):107117. [PubMed: 15668327]

10. Habermann JK, Paulsen U, Roblick UJ, et al. Stage-specific alterations of the genome, transcriptome, and proteome during colorectal carcinogenesis. Genes Chromosomes Cancer. 2007; 46(1):10-26. [PubMed: 17044061]

11. Selvarajah S, Yoshimoto M, Ludkovski O, et al. Genomic signatures of chromosomal instability and osteosarcoma progression detected by high resolution array CGH and interphase FISH. Cytogenet Genome Res. 2008; 122(1):5-15. [PubMed: 18931480]

12. Heilig CE, Loffler H, Mahlknecht U, et al. Chromosomal instability correlates with poor outcome in patients with myelodysplastic syndromes irrespectively of the cytogenetic risk group. J Cell Mol Med. 2010; 14(4):895-902. [PubMed: 19754665]

13. Hart JR, Glebov O, Ernst RJ, et al. DNA mismatch-specific targeting and hypersensitivity of mismatch-repair-deficient cells to bulky rhodium(III) intercalators. Proc Natl Acad Sci U S A. 2006; 103(42):15359-15363. [PubMed: 17030786]

14. Junicke H, Hart JR, Kisko J, et al. A rhodium(III) complex for high-affinity DNA base-pair mismatch recognition. Proc Natl Acad Sci U S A. 2003; 100(7):3737-3742. [PubMed: 12610209]

15. Pierre VC, Kaiser JT, Barton JK. Insights into finding a mismatch through the structure of a mispaired DNA bound by a rhodium intercalator. Proc Natl Acad Sci U S A. 2007; 104(2):429434. [PubMed: 17194756]

16. Ernst RJ, Song H, Barton JK. DNA mismatch binding and antiproliferative activity of rhodium metalloinsertors. J Am Chem Soc. 2009; 131(6):2359-2366. [PubMed: 19175313]

17. Torres EM, Williams BR, Amon A. Aneuploidy: cells losing their balance. Genetics. 2008; 179(2): 737-746. [PubMed: 18558649]

18. Lindsley DL, Sandler L, Baker BS, et al. Segmental aneuploidy and the genetic gross structure of the Drosophila genome. Genetics. 1972; 71(1):157-184. [PubMed: 4624779]

19. Hassold TJ, Jacobs PA. Trisomy in man. Annu Rev Genet. 1984; 18:69-97. [PubMed: 6241455]

20. Segal DJ, McCoy EE. Studies on Down's syndrome in tissue culture. I. Growth rates and protein contents of fibroblast cultures. J Cell Physiol. 1974; 83(1):85-90. [PubMed: 4273197]

21. Williams BR, Prabhu VR, Hunter KE, et al. Aneuploidy affects proliferation and spontaneous immortalization in mammalian cells. Science. 2008; 322(5902):703-709. [PubMed: 18974345]

22. Torres EM, Sokolsky T, Tucker CM, et al. Effects of aneuploidy on cellular physiology and cell division in haploid yeast. Science. 2007; 317(5840):916-924. [PubMed: 17702937]

23. Niwa O, Tange Y, Kurabayashi A. Growth arrest and chromosome instability in aneuploid yeast. Yeast. 2006; 23(13):937-950. [PubMed: 17072887]

24. Ganmore I, Smooha G, Izraeli S. Constitutional aneuploidy and cancer predisposition. Hum Mol Genet. 2009; 18(R1):R84-R93. [PubMed: 19297405]

25. Ried T, Knutzen R, Steinbeck R, et al. Comparative genomic hybridization reveals a specific pattern of chromosomal gains and losses during the genesis of colorectal tumors. Genes Chromosomes Cancer. 1996; 15(4):234-245. [PubMed: 8703849] 
26. Rooney PH, Murray GI, Stevenson DA, et al. Comparative genomic hybridization and chromosomal instability in solid tumours. Br J Cancer. 1999; 80(5-6):862-873. [PubMed: 10360667]

27. Kallioniemi A. CGH microarrays and cancer. Curr Opin Biotechnol. 2008; 19(1):36-40. [PubMed: 18162393]

28. Tsukamoto Y, Uchida T, Karnan S, et al. Genome-wide analysis of DNA copy number alterations and gene expression in gastric cancer. J Pathol. 2008; 216(4):471-482. [PubMed: 18798223]

29. Carter SL, Eklund AC, Kohane IS, et al. A signature of chromosomal instability inferred from gene expression profiles predicts clinical outcome in multiple human cancers. Nat Genet. 2006; 38(9): 1043-1048. [PubMed: 16921376]

30. Swanton C, Nicke B, Schuett M, et al. Chromosomal instability determines taxane response. Proc Natl Acad Sci U S A. 2009; 106(21):8671-8676. [PubMed: 19458043]

31. Sudo T, Nitta M, Saya H, et al. Dependence of paclitaxel sensitivity on a functional spindle assembly checkpoint. Cancer Res. 2004; 64(7):2502-2508. [PubMed: 15059905]

32. Swanton C, Marani M, Pardo O, et al. Regulators of mitotic arrest and ceramide metabolism are determinants of sensitivity to paclitaxel and other chemotherapeutic drugs. Cancer Cell. 2007; 11(6):498-512. [PubMed: 17560332]

33. Upender MB, Habermann JK, McShane LM, et al. Chromosome transfer induced aneuploidy results in complex dysregulation of the cellular transcriptome in immortalized and cancer cells. Cancer Res. 2004; 64(19):6941-6949. [PubMed: 15466185]

34. Weaver BA, Silk AD, Montagna C, et al. Aneuploidy acts both oncogenically and as a tumor suppressor. Cancer Cell. 2007; 11(1):25-36. [PubMed: 17189716]

35. Williams BR, Amon A. Aneuploidy: cancer's fatal flaw? Cancer Res. 2009; 69(13):5289-5891. [PubMed: 19549887]

36. Hartwell L. Defects in a cell cycle checkpoint may be responsible for the genomic instability of cancer cells. Cell. 1992; 71(4):543-546. [PubMed: 1423612]

37. Loeb LA. Mutator phenotype may be required for multistage carcinogenesis. Cancer Res. 1991; 51(12):3075-3079. [PubMed: 2039987]

38. Heppner GH. Tumor heterogeneity. Cancer Res. 1984; 44(6):2259-2265. [PubMed: 6372991]

39. Lengauer C, Kinzler KW, Vogelstein B. Genetic instability in colorectal cancers. Nature. 1997; 386(6625):623-627. [PubMed: 9121588]

40. Lengauer C, Kinzler KW, Vogelstein B. Genetic instabilities in human cancers. Nature. 1998; 396(6712):643-649. [PubMed: 9872311]

41. Bayani J, Selvarajah S, Maire G, et al. Genomic mechanisms and measurement of structural and numerical instability in cancer cells. Semin Cancer Biol. 2007; 17(1):5-18. [PubMed: 17126026]

42. Roschke AV, Stover K, Tonon G, et al. Stable karyotypes in epithelial cancer cell lines despite high rates of ongoing structural and numerical chromosomal instability. Neoplasia. 2002; 4(1):1931. [PubMed: 11922387]

43. Camps J, Ponsa I, Ribas M, et al. Comprehensive measurement of chromosomal instability in cancer cells: combination of fluorescence in situ hybridization and cytokinesis-block micronucleus assay. FASEB J. 2005; 19(7):828-830. [PubMed: 15760839]

44. Gao C, Furge K, Koeman J, et al. Chromosome instability, chromosome transcriptome, and clonal evolution of tumor cell populations. Proc Natl Acad Sci U S A. 2007; 104(21):8995-9000. [PubMed: 17517657]

45. Holland AJ, Cleveland DW. Boveri revisited: chromosomal instability, aneuploidy and tumorigenesis. Nat Rev Mol Cell Biol. 2009; 10(7):478-487. [PubMed: 19546858]

46. Foijer F, Draviam VM, Sorger PK. Studying chromosome instability in the mouse. Biochim Biophys Acta. 2008; 1786(1):73-82. [PubMed: 18706976]

47. Rao CV, Yamada HY, Yao Y, et al. Enhanced genomic instabilities caused by deregulated microtubule dynamics and chromosome segregation: a perspective from genetic studies in mice. Carcinogenesis. 2009; 30(9):1469-1474. [PubMed: 19372138]

48. Weaver BA, Cleveland DW. The role of aneuploidy in promoting and suppressing tumors. J Cell Biol. 2009; 185(6):935-937. [PubMed: 19528293] 
49. Iwanaga Y, Chi YH, Miyazato A, et al. Heterozygous deletion of mitotic arrest-deficient protein 1 (MAD1) increases the incidence of tumors in mice. Cancer Res. 2007; 67(1):160-166. [PubMed: 17210695]

50. Kalitsis P, Earle E, Fowler KJ, et al. Bub3 gene disruption in mice reveals essential mitotic spindle checkpoint function during early embryogenesis. Genes Dev. 2000; 14(18):2277-2282. [PubMed: 10995385]

51. Michel LS, Liberal V, Chatterjee A, et al. MAD2 haploinsufficiency causes premature anaphase and chromosome instability in mammalian cells. Nature. 2001; 409(6818):355-359. [PubMed: 11201745]

52. Perera D, Tilston V, Hopwood JA, et al. Bub1 maintains centromeric cohesion by activation of the spindle checkpoint. Dev Cell. 2007; 13(4):566-579. [PubMed: 17925231]

53. Putkey FR, Cramer T, Morphew MK, et al. Unstable kinetochore-microtubule capture and chromosomal instability following deletion of CENP-E. Dev Cell. 2002; 3(3):351-365. [PubMed: 12361599]

54. Baker DJ, Jeganathan KB, Cameron JD, et al. BubR1 insufficiency causes early onset of agingassociated phenotypes and infertility in mice. Nat Genet. 2004; 36(7):744-749. [PubMed: 15208629]

55. Jeganathan K, Malureanu L, Baker DJ, et al. Bub1 mediates cell death in response to chromosome missegregation and acts to suppress spontaneous tumorigenesis. J Cell Biol. 2007; 179(2):255267. [PubMed: 17938250]

56. Jeganathan KB, Baker DJ, van Deursen JM. Securin associates with APCCdh1 in prometaphase but its destruction is delayed by Rae1 and Nup98 until the metaphase/anaphase transition. Cell Cycle. 2006; 5(4):366-370. [PubMed: 16479161]

57. Babu JR, Jeganathan KB, Baker DJ, et al. Rae1 is an essential mitotic checkpoint regulator that cooperates with Bub3 to prevent chromosome missegregation. J Cell Biol. 2003; 160(3):341-353. [PubMed: 12551952]

58. Dai W, Wang Q, Liu T, et al. Slippage of mitotic arrest and enhanced tumor development in mice with BubR1 haploinsufficiency. Cancer Res. 2004; 64(2):440-445. [PubMed: 14744753]

59. Weaver BA, Cleveland DW. Aneuploidy: instigator and inhibitor of tumorigenesis. Cancer Res. 2007; 67(21):10103-10105. [PubMed: 17974949]

60. Weaver BA, Silk AD, Cleveland DW. Low rates of aneuploidy promote tumorigenesis while high rates of aneuploidy cause cell death and tumor suppression. Cell Oncol. 2008; 30(5):453. [PubMed: 18791276]

61. Baker DJ, Jin F, Jeganathan KB, et al. Whole chromosome instability caused by Bub1 insufficiency drives tumorigenesis through tumor suppressor gene loss of heterozygosity. Cancer Cell. 2009; 16(6):475-486. [PubMed: 19962666]

62. Wang X, Cheung HW, Chun AC, et al. Mitotic checkpoint defects in human cancers and their implications to chemotherapy. Front Biosci. 2008; 13:2103-2114. [PubMed: 17981695]

63. Barber TD, McManus K, Yuen KW, et al. Chromatid cohesion defects may underlie chromosome instability in human colorectal cancers. Proc Natl Acad Sci U S A. 2008; 105(9):3443-3448. [PubMed: 18299561]

64. Yu R, Lu W, Chen J, et al. Overexpressed pituitary tumor-transforming gene causes aneuploidy in live human cells. Endocrinology. 2003; 144(11):4991-4998. [PubMed: 12960092]

65. Zhang N, Ge G, Meyer R, et al. Overexpression of Separase induces aneuploidy and mammary tumorigenesis. Proc Natl Acad Sci U S A. 2008; 105(35):13033-13038. [PubMed: 18728194]

66. Brinkley BR. Managing the centrosome numbers game: from chaos to stability in cancer cell division. Trends Cell Biol. 2001; 11(1):18-21. [PubMed: 11146294]

67. Nigg EA. Centrosome aberrations: cause or consequence of cancer progression? Nat Rev Cancer. 2002; 2(11):815-825. [PubMed: 12415252]

68. Ring D, Hubble R, Kirschner M. Mitosis in a cell with multiple centrioles. J Cell Biol. 1982; 94(3): 549-556. [PubMed: 7130271]

69. Ganem NJ, Godinho SA, Pellman D. A mechanism linking extra centrosomes to chromosomal instability. Nature. 2009; 460(7252):278-282. [PubMed: 19506557] 
70. Silkworth WT, Nardi IK, Scholl LM, et al. Multipolar spindle pole coalescence is a major source of kinetochore mis-attachment and chromosome mis-segregation in cancer cells. PLoS One. 2009; 4(8):e6564. [PubMed: 19668340]

71. Cimini D. Merotelic kinetochore orientation, aneuploidy, and cancer. Biochim Biophys Acta. 2008; 1786(1):32-40. [PubMed: 18549824]

72. Cimini D. Detection and correction of merotelic kinetochore orientation by Aurora B and its partners. Cell Cycle. 2007; 6(13):1558-1564. [PubMed: 17603301]

73. Cimini D, Wan X, Hirel CB, et al. Aurora kinase promotes turnover of kinetochore microtubules to reduce chromosome segregation errors. Curr Biol. 2006; 16(17):1711-1718. [PubMed: 16950108]

74. Bakhoum SF, Genovese G, Compton DA. Deviant kinetochore microtubule dynamics underlie chromosomal instability. Curr Biol. 2009; 19(22):1937-1942. [PubMed: 19879145]

75. Thompson SL, Compton DA. Examining the link between chromosomal instability and aneuploidy in human cells. J Cell Biol. 2008; 180(4):665-672. [PubMed: 18283116]

76. Bakhoum SF, Thompson SL, Manning AL, et al. Genome stability is ensured by temporal control of kinetochore-microtubule dynamics. Nat Cell Biol. 2009; 11(1):27-35. [PubMed: 19060894]

77. Bakhoum SF, Compton DA. Cancer: CINful centrosomes. Curr Biol. 2009; 19(15):R642-R645. [PubMed: 19674544]

78. McClelland SE, Burrell RA, Swanton C. Chromosomal instability: a composite phenotype that influences sensitivity to chemotherapy. Cell Cycle. 2009; 8(20):3262-3266. [PubMed: 19806022]

79. Roschke AV, Glebov OK, Lababidi S, et al. Chromosomal instability is associated with higher expression of genes implicated in epithelial-mesenchymal transition, cancer invasiveness, and metastasis and with lower expression of genes involved in cell cycle checkpoints, DNA repair, and chromatin maintenance. Neoplasia. 2008; 10(11):1222-1230. [PubMed: 18953431]

80. Kwon M, Godinho SA, Chandhok NS, et al. Mechanisms to suppress multipolar divisions in cancer cells with extra centrosomes. Genes Dev. 2008; 22(16):2189-2203. [PubMed: 18662975]

81. Mountain V, Simerly C, Howard L, et al. The kinesin-related protein, HSET, opposes the activity of Eg5 and cross-links microtubules in the mammalian mitotic spindle. J Cell Biol. 1999; 147(2): 351-366. [PubMed: 10525540]

82. Chen JG, Horwitz SB. Differential mitotic responses to microtubule-stabilizing and -destabilizing drugs. Cancer Res. 2002; 62(7):1935-1938. [PubMed: 11929805]

83. Rebacz B, Larsen TO, Clausen MH, et al. Identification of griseofulvin as an inhibitor of centrosomal clustering in a phenotype-based screen. Cancer Res. 2007; 67(13):6342-6350. [PubMed: 17616693]

84. Ronnest MH, Rebacz B, Markworth L, et al. Synthesis and structure-activity relationship of griseofulvin analogues as inhibitors of centrosomal clustering in cancer cells. J Med Chem. 2009; 52(10):3342-3347. [PubMed: 19402668]

85. Roschke AV, Lababidi S, Tonon G, et al. Karyotypic "state" as a potential determinant for anticancer drug discovery. Proc Natl Acad Sci U S A. 2005; 102(8):2964-2969. [PubMed: 15703300]

86. Wallqvist A, Huang R, Covell DG, et al. Drugs aimed at targeting characteristic karyotypic phenotypes of cancer cells. Mol Cancer Ther. 2005; 4(10):1559-1568. [PubMed: 16227406]

87. Roschke AV, Kirsch IR. Targeting cancer cells by exploiting karyotypic complexity and chromosomal instability. Cell Cycle. 2005; 4(5):679-682. [PubMed: 15846096]

88. Rabow AA, Shoemaker RH, Sausville EA, et al. Mining the National Cancer Institute's tumorscreening database: identification of compounds with similar cellular activities. J Med Chem. 2002; 45(4):818-840. [PubMed: 11831894]

89. Covell DG, Wallqvist A, Huang R, et al. Linking tumor cell cytotoxicity to mechanism of drug action: an integrated analysis of gene expression, small-molecule screening and structural databases. Proteins. 2005; 59(3):403-433. [PubMed: 15778971]

90. Huang R, Wallqvist A, Thanki N, et al. Linking pathway gene expressions to the growth inhibition response from the National Cancer Institute's anticancer screen and drug mechanism of action. Pharmacogenom J. 2005; 5(6):381-399. 
91. Duncan KL, Duncan MD, Alley MC, et al. Cucurbitacin E-induced disruption of the actin and vimentin cytoskeleton in prostate carcinoma cells. Biochem Pharmacol. 1996; 52(10):1553-1560. [PubMed: 8937470]

92. Duncan MD, Duncan KL. Cucurbitacin E targets proliferating endothelia. J Surg Res. 1997; 69(1): 55-60. [PubMed: 9202647]

93. Sun J, Blaskovich MA, Jove R, et al. Cucurbitacin Q: a selective STAT3 activation inhibitor with potent antitumor activity. Oncogene. 2005; 24(20):3236-3245. [PubMed: 15735720]

94. Andreassen PR, Martineau SN, Margolis RL. Chemical induction of mitotic checkpoint override in mammalian cells results in aneuploidy following a transient tetraploid state. Mutat Res. 1996; 372(2):181-194. [PubMed: 9015137]

95. Cha SK, Kim NH, Lee SM, et al. Effect of cytochalasin B and cycloheximide on the activation rate, chromosome constituent and in vitro development of porcine oocytes following parthenogenetic stimulation. Reprod Fertil Dev. 1997; 9(4):441-446. [PubMed: 9402254]

96. Scala S, Akhmed N, Rao US, et al. P-glycoprotein substrates and antagonists cluster into two distinct groups. Mol Pharmacol. 1997; 51(6):1024-1033. [PubMed: 9187269]

97. Wosikowski K, Schuurhuis D, Johnson K, et al. Identification of epidermal growth factor receptor and c-erbB2 pathway inhibitors by correlation with gene expression patterns. J Natl Cancer Inst. 1997; 89(20):1505-1515. [PubMed: 9337347]

98. Gupta AK, Misra HK. Synthesis and evaluation of substituted quinazolone derivatives for antibacterial, antifungal, and antiacetylcholinesterase activities. J Pharm Sci. 1980; 69(11):13131317. [PubMed: 7452462]

99. Sakowicz R, Finer JT, Beraud C, et al. Antitumor activity of a kinesin inhibitor. Cancer Res. 2004; 64(9):3276-3280. [PubMed: 15126370]

100. Ferrari S, Costi PM, Wade RC. Inhibitor specificity via protein dynamics: insights from the design of antibacterial agents targeted against thymidylate synthase. Chem Biol. 2003; 10(12): 1183-1193. [PubMed: 14700626] 

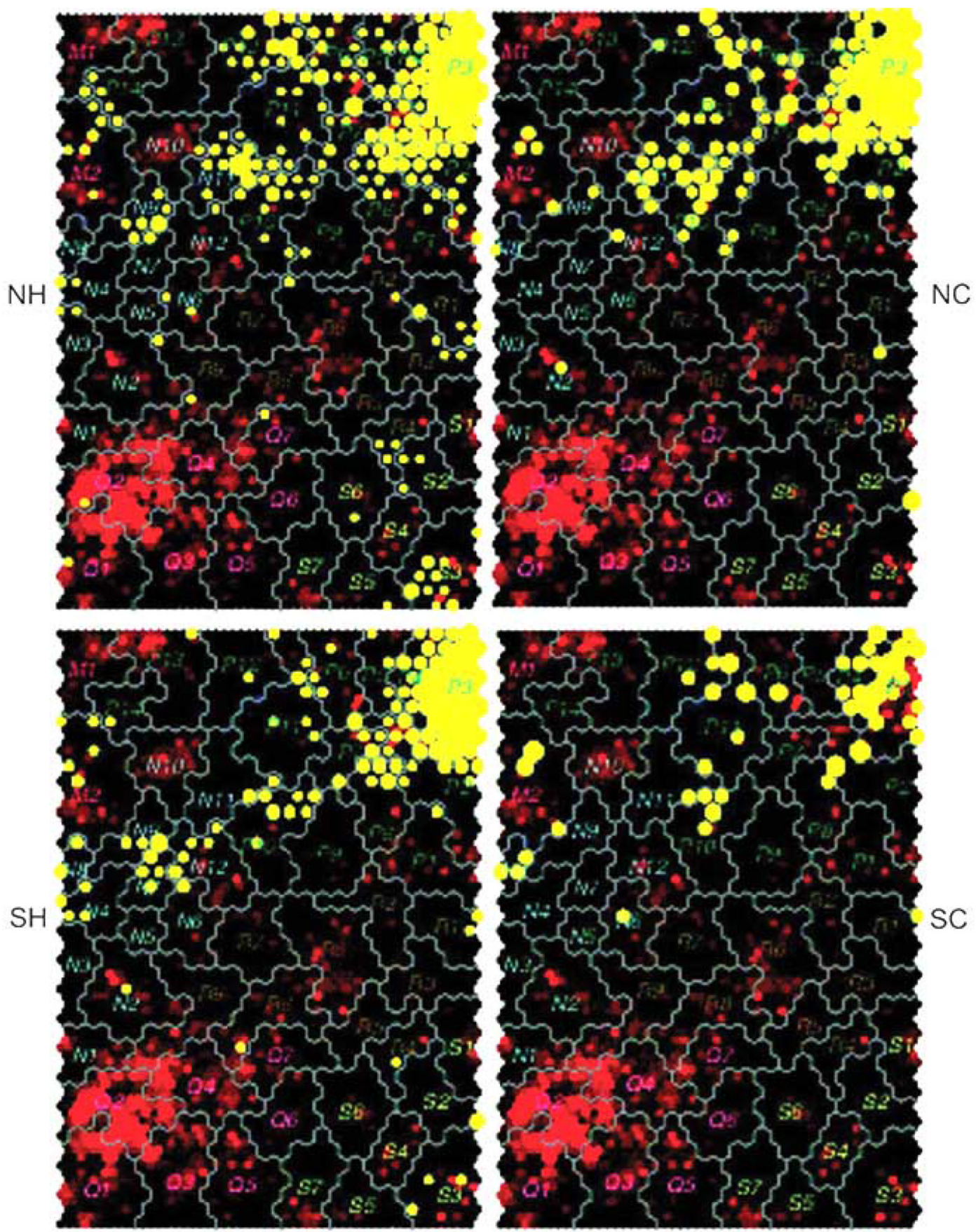


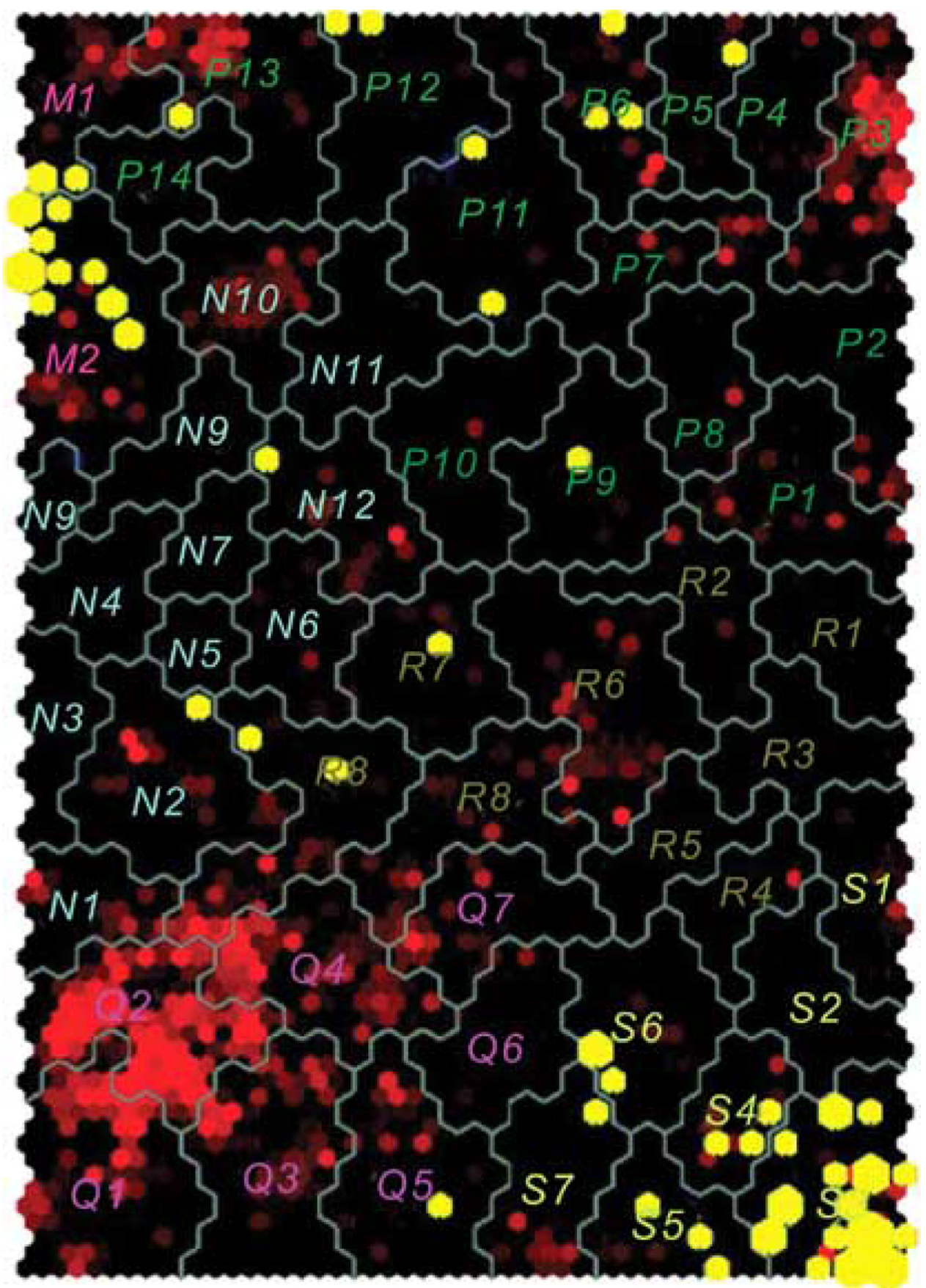

Fig. (1).

a. Projection of positively correlated $\mathrm{GI}_{50}$ vectors with karyotypic variables. At the selected $\mathrm{P}$ of 0.05 , the karyotypic variables are correlated with $1,198,1,006,1,271$, and $381 \mathrm{GI}_{50}$ data vectors for numerical heterogeneity $(\mathrm{NH})$, numerical complexity $(\mathrm{NC})$, structural heterogeneity $(\mathrm{SH})$, and structural complexity (SC), respectively, of which the top 500 hits (yellow dots) are shown (Wallqvist et al., 2005).

b. Projection of $\mathrm{GI}_{50}$ data vectors for drugs in clinical use as anticancer agents (Wallqvist et al., 2005). 
<smiles>CC(=O)OC(C)(C)/C=C\C(=O)C(C)(O)C1C(O)CC2(C)C3CC=C4C(C=C(O)C(=O)C4(C)C)C3(C)C(=O)CC12C</smiles>

A. Cucurbitacins

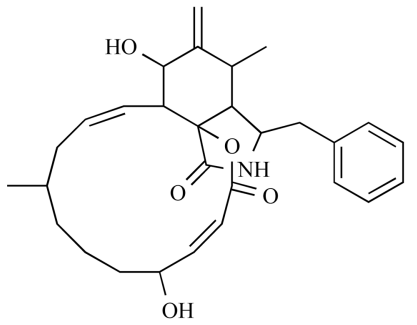

B. Cytochalasins

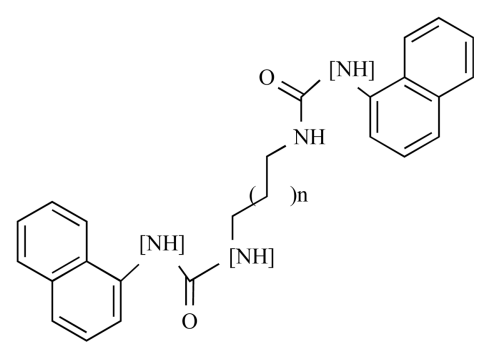

C. Bis-naphthylcarboxamides and Bis-napthylureas<smiles>COc1ccc(NC(=O)CC(=O)n2nc(-c3ccccc3)c(/N=N/c3ccccc3)c2-c2ccccc2)cc1</smiles>

D. Anilinomalonyl phenylazopyrazoles<smiles>N#Cc1c(-c2ccccc2)ccn(C2OCC(O)C(O)C2O)c1=S</smiles>

E. Pyridinethione carbonitrile nucleosides

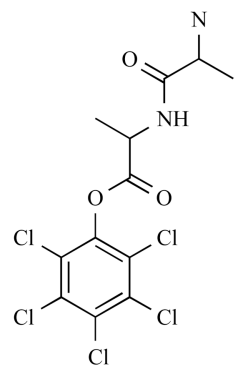

F. Pentachlorophenyl polypeptide esters

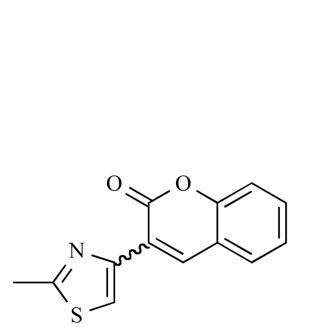

G. Thiazolyl coumarins<smiles>Cc1nc2ccc(C(C)(C)C)cc2nc1NCc1ccccc1</smiles>

H. Anilino/phenoxy-carboxy/phenyl-6(7)substituted quinooxalines<smiles>CC=C1CC(CO)(COC(C)=O)OC1=O</smiles>

J. 3-Alkylidene-5,5-disubstituted tetrahydro-2-furanones<smiles>CSc1nc2ccc([AlH2])cc2c(=O)n1Cc1ccccc1</smiles>

K. 2-Substituted mercapto$3 \mathrm{H}$-quinazolines

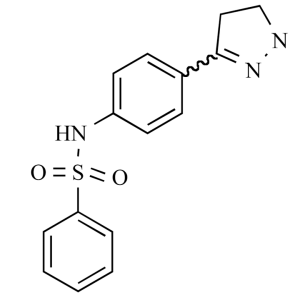

L. N-(p-(substituted azole)phenyl) benzenesulfonamides

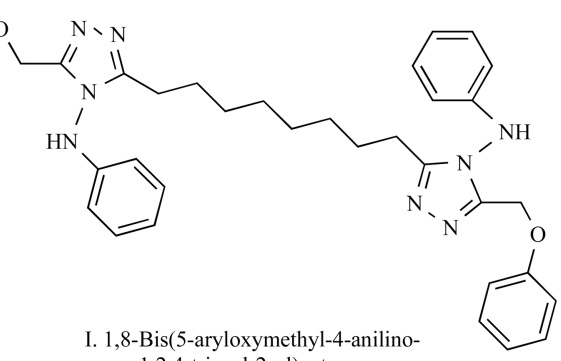
1,2,4-triazol-2-yl)octanes

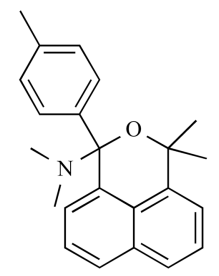

M. 1,1-Dimethyl-3phenyl-3-pyrrolidinyl/4morpholinyl napthalans

Fig. (2).

Structural motifs identified as positively correlated with karyotypic variables (Wallqvist et al., 2005). 
Table 1

Groups of Compounds more Active Against Cell Lines with Complex and/or Heterogeneous Karyotypes (Roschke et al., 2005)

\begin{tabular}{|c|c|}
\hline Groups of compounds & $\begin{array}{c}\text { Number of } \\
\text { compounds in } \\
\text { the group }\end{array}$ \\
\hline Ellipticine/Ellipticinium/Olivacinium group & 10 \\
\hline Fuchsine group & 3 \\
\hline Cytochalasin group & 3 \\
\hline 2-propenamide,N-[variable]-3-(1,2,3,4,tetrahydro-6-methyl-2,4-dioxo-5-pyrimidinyl) group & 6 \\
\hline Benzodithiophene-4,8-dione group & 6 \\
\hline Combretastatin group & 4 \\
\hline Antibiotics & 13 \\
\hline Others & 8 \\
\hline Total \# of compounds: & 53 \\
\hline
\end{tabular}

